\title{
Expression Profile of Genes Potentially Associated with Adequate Glycemic Control in Patients with Type 2 Diabetes Mellitus
}

\author{
Sâmia Cruz Tfaile Corbi, ${ }^{1,2}$ Alliny Souza Bastos, ${ }^{1}$ Rafael Nepomuceno, ${ }^{1,2}$ \\ Thamiris Cirelli, ${ }^{1,2}$ Raquel Alves dos Santos, ${ }^{3}$ Catarina Satie Takahashi, ${ }^{4}$ \\ Cristiane S. Rocha, ${ }^{5}$ Silvana Regina Perez Orrico, ${ }^{1}$ Claudia V. Maurer-Morelli, ${ }^{5}$ and \\ Raquel Mantuaneli Scarel-Caminaga ${ }^{2}$ \\ ${ }^{1}$ Department of Diagnosis and Surgery, School of Dentistry, Universidade Estadual Paulista (UNESP), Araraquara, SP, Brazil \\ ${ }^{2}$ Department of Morphology, School of Dentistry, Universidade Estadual Paulista (UNESP), Araraquara, SP, Brazil \\ ${ }^{3}$ Postgraduate Program in Sciences of the University of Franca, Franca, SP, Brazil \\ ${ }^{4}$ Department of Genetics, Faculty of Medicine of Ribeirão Preto and Department of Biology, FFCLRP, University of São Paulo (USP), \\ Ribeirão Preto, SP, Brazil \\ ${ }^{5}$ Department of Medical Genetics, University of Campinas (UNICAMP), Campinas, SP, Brazil
}

Correspondence should be addressed to Raquel Mantuaneli Scarel-Caminaga; raquel@foar.unesp.br

Received 16 April 2017; Revised 22 May 2017; Accepted 31 May 2017; Published 25 July 2017

Academic Editor: Hiroshi Okamoto

Copyright @ 2017 Sâmia Cruz Tfaile Corbi et al. This is an open access article distributed under the Creative Commons Attribution License, which permits unrestricted use, distribution, and reproduction in any medium, provided the original work is properly cited.

\begin{abstract}
Despite increasing research in type 2 diabetes mellitus (T2D), there are few studies showing the impact of the poor glycemic control on biological processes occurring in T2D. In order to identify potential genes related to poorly/well-controlled patients with T2D, our strategy of investigation included a primary screen by microarray (Human Genome U133) in a small group of individuals followed by an independent validation in a greater group using RT-qPCR. Ninety patients were divided as follows: poorly controlled T2D (G1), well-controlled T2D (G2), and normoglycemic individuals (G3). After using affy package in R, differentially expressed genes (DEGs) were prospected as candidate genes potentially relevant for the glycemic control in T2D patients. After validation by RT-qPCR, the obtained DEGs were as follows-G1 + G2 versus G3: HLA-DQA1, SOS1, and BRCA2; G2 versus G1: ENO2, VAMP2, CCND3, CEBPD, LGALS12, AGBL5, MAP2K5, and PPAP2B; G2 versus G3: HLA-DQB1, MCM4, and SEC13; and G1 versus G3: PPIC. This demonstrated a systemic exacerbation of the gene expression related to immune response in T2D patients. Moreover, genes related to lipid metabolisms and DNA replication/repair were influenced by the glycemic control. In conclusion, this study pointed out candidate genes potentially associated with adequate glycemic control in T2D patients, contributing to the knowledge of how the glycemic control could systemically influence gene expression.
\end{abstract}

\section{Introduction}

Diabetes mellitus (DM), characterized by hyperglycemia, is associated with the progressive development of complications, like atherosclerosis, renal and neuronal damage, and blindness [1]. Type 2 diabetes mellitus (T2D), the most common form of diabetes mellitus (DM) accounting for $80-90 \%$ of the cases, is a chronic polygenic disorder identified by defects in insulin action and/or deficiencies in pancreatic insulin secretion [2, 3]. It is known that T2D occurs concomitantly to other systemic diseases, such as dyslipidemia [4] and cardiovascular diseases [5]. Patients affected by these diseases have demonstrated a hyperinflammatory state [6-8] that put their health at major risk $[9,10]$. It has been shown that chronically elevated levels of inflammatory markers in T2D patients with poor glycemic control can increase the risk for myocardial infarction [11] and infectious diseases, including chronic periodontitis [12].

Adequate glycemic control remains a challenge for clinicians; thus, more studies are needed to investigate the impact 
of poor glycemic control on diverse biological mechanistic processes in patients with T2D. It is already known that hyperglycemia can lead to vascular complications via several mechanisms, and clinical trials have reported that intensive glycemic control significantly reduces microvascular complications, for instance, retinopathy, nephropathy, and peripheral arterial disease [13-15].

A significant difference in the expression of genes involved in insulin signaling and immune response, for example, TNF $\alpha$, interleukin- (IL-) 6, and IL-1 $\beta$, was found in PBMCs from T2DM and impaired glucose tolerance patients [16-18]. The influence of hyperglycemia on gene expression, somewhat, can explain the distinct expression profiles displayed by diabetic patients compared to the control group, such as fatty acid metabolism and protection against lipid-induced oxidative stress, adipocytokine, and ceramide signaling pathways [16].

Despite previous reports, adequate glycemic control remains a challenge for clinicians; thus, additional studies are necessary to investigate the impact of poor glycemic control on patients with T2D. Therefore, we utilized a primary microarray screening to prospect candidate genes potentially relevant for the glycemic control in T2D patients, and to validate this finding, we investigated their expression levels in T2D patients with poor or good glycemic control and in normoglycemic individuals.

\section{Materials and Methods}

2.1. Study Population and Physical and Biochemical Evaluations. This study was approved by the Ethics in Human Research Committee of School of Dentistry (Universidade Estadual Paulista (UNESP), Araraquara, Brazil; Protocol number 50/06) and was conducted according to the ethical principles of the Declaration of Helsinki. During three years (2009-2011), after we examined 1788 patients, we selected 90 patients according to our criteria of inclusion/ exclusion as detailed in our previous studies [19-21]. From the selected patients, blood samples were collected after a 12-hour overnight fast for the evaluation of fasting plasma glucose $(\mathrm{mg} / \mathrm{dl})$, glycated hemoglobin (HbAlc), and insulin levels $(\mathrm{U} / \mathrm{l})$, in which all the analyses were performed by the same laboratory. Physical examinations including measurement of waist and hip circumference (centimeters), height (meters), weight (kilograms), and body mass index (BMI) were evaluated in all selected patients.

Based upon diabetic and glycemic control statuses, monitored by an endocrinologist, 90 patients were enrolled in this study who were divided into three groups $(G)$ of 30 patients each as follows: poorly controlled T2D (G1, HbA1c $\geq 8.5 \%$ ), well-controlled T2D (G2, HbA1c $<7.0 \%)$, and normoglycemic individuals (G3, HbA1c<6.5\%) [19]. Patients from G1, G2, and G3 were tightly matched for the presence of two common comorbidities: dyslipidemia and chronic periodontitis, as previously demonstrated in Bastos et al. [20] and Corbi et al. [21].

2.2. Selection of Candidate Genes. We used a list of differentially expressed genes (DEGs) based on microarray analysis
(Human U133 Plus 2.0 Affymetrix Inc., Santa Clara, CA, USA - unpublished data) to select candidate genes in poorly and well-controlled T2D as well as normoglycemic patients. Microarray data was generated from patients of G1 $(n=5)$, G2 $(n=7)$, and G3 $(n=6)$, after considering greater homogeneity regarding biochemical parameters. DEGs were selected as follows: G1 + G2 versus G3, in order to compare T2D patients (independent of glycemic control) with normoglycemic individuals; G2 versus G1, to access the effect of good glycemic control on the gene expression, in comparison with poorly controlled T2D patients; G2 versus G3, to access the effect of good glycemic control on the T2D patients in comparison with normoglycemic individuals; and G1 versus G3, to access the effect of poor glycemic control on the T2D patients in comparison with normoglycemic individuals.

\subsection{Reverse Transcription-Quantitative Polymerase Chain} Reaction (RT-qPCR) Real-Time Analysis. For this study, each group was composed of 30 patients (including patients chosen for microarray analysis) totalizing 90 patients. Reverse transcription reactions for the complementary DNA (cDNA) were made utilizing the SuperScript III First Strand Synthesis SuperMix (Invitrogen). Considering together the three studied groups, three genes were identified by microarray data as demonstrating the best housekeeping expression patterns: ACTG1 (Hs03044422_g1), RPL7A (Hs00605223_g1), and GAPDH (Hs02758991_g1). The mean values of them were used as endogenous controls of the qPCR reactions. All reactions were performed in duplicate utilizing TaqMan ${ }^{\circledR}$ gene expression assays (Applied Biosystems) in the 7500 RealTime PCR System (Applied Biosystems, Foster City, CA, USA). PCR cycling parameters were 2 minutes at $50^{\circ} \mathrm{C}, 10$ minutes at $95^{\circ} \mathrm{C}$ and 40 cycles of 15 seconds at $95^{\circ} \mathrm{C}$, and 60 seconds at $60^{\circ} \mathrm{C}$. To calculate gene expression, the Expression Suite Software was used (Applied Biosystems, Foster City, CA, USA), which employs the comparative $\mathrm{C} \tau(\Delta \mathrm{C} \tau)$ method for multiplate data analysis.

2.4. Statistical Analysis. General characteristics of each group were described by mean and standard deviation (SD). The distribution and normality of the demographic and clinical variables were evaluated by the D'Agostino-Pearson test. Accordingly, we used the chi-squared test (for gender), and to compare other characteristics among the three groups, we used the Kruskal-Wallis test (followed by Dunn's posttest), alternatively, when only two groups were compared.

For RT-qPCR analyses, values of $2^{-\Delta C \tau}$ were compared between each two groups by the Mann-Whitney test using a significance level of $p=0.05$. These analyses were carried out in the GraphPad Prism software, version 5.0.

\section{Results}

3.1. Sample Population. Table 1 shows demographic, physical, and biochemical data of the investigated patients. Gender, age, ethnicity, and socioeconomic status were similar among the three groups. Independent of the group, all individuals were overweight, and the diabetic patients were obese and presented higher values of BMI, abdominal circumference, 
TABLE 1: Characteristics of the sample: demographic, physical, biochemical, and diabetic data (mean $\pm \mathrm{SD}$ ).

\begin{tabular}{|c|c|c|c|}
\hline & $\begin{array}{c}\text { Group } 1 \\
n=30\end{array}$ & $\begin{array}{c}\text { Group } 2 \\
n=30\end{array}$ & $\begin{array}{c}\text { Group } 3 \\
n=30\end{array}$ \\
\hline Gender (F/M) & $18 / 12$ & $20 / 10$ & $17 / 13$ \\
\hline Age $($ mean $\pm S D)$ & $48.0( \pm 7.6)$ & $50.3( \pm 6.7)$ & $49.0( \pm 7.5)$ \\
\hline Ethnicity (white/brown/black) & $13 / 10 / 7$ & $15 / 11 / 4$ & $20 / 8 / 2$ \\
\hline $\mathrm{BMI}\left(\mathrm{m} / \mathrm{kg}^{2}\right)$ & $30.5( \pm 5.2)$ & $31.4( \pm 4.1)$ & $28.4( \pm 3.8)$ \\
\hline Abdominal circumference $(\mathrm{cm})^{*}$ & $104.3( \pm 14.6)$ & $109.3( \pm 10.8)^{\mathrm{a}}$ & $98.1( \pm 9.9)$ \\
\hline Waist/hip proportion & $1.0( \pm 0.1)$ & $1.0( \pm 0.1)$ & $0.9( \pm 0.1)$ \\
\hline Fasting glucose $(\mathrm{mg} / \mathrm{dl})^{*}$ & $226.6( \pm 74.2)^{\mathrm{a}, \mathrm{b}}$ & $137.5( \pm 41.4)^{\mathrm{a}}$ & $90.0( \pm 6.4)$ \\
\hline $\operatorname{HbAlc}(\%)^{*}$ & $10.4( \pm 1.9)^{\mathrm{a}, \mathrm{b}}$ & $6.6( \pm 0.9)^{\mathrm{a}}$ & $5.4( \pm 0.6)$ \\
\hline Insulin $(\mathrm{U} / \mathrm{l})^{*}$ & $19.7( \pm 20.9)^{\mathrm{a}}$ & $21.1( \pm 21.5)^{\mathrm{a}}$ & $12.6( \pm 8.5)$ \\
\hline $\begin{array}{l}\text { Time since DM onset } \\
\text { (years) }(\text { mean } \pm S D)\end{array}$ & $6.2( \pm 4.2)$ & $5.2( \pm 6.6)$ & - \\
\hline \multicolumn{4}{|l|}{ Presence of diabetes } \\
\hline Total complications ${ }^{c}$ & $19^{c}$ & 12 & - \\
\hline \multicolumn{4}{|l|}{ Medication for DM control } \\
\hline Hypoglycemic & 20 & 15 & - \\
\hline Insulin & 1 & 1 & - \\
\hline Hypoglycemic/insulin & 8 & 5 & - \\
\hline None & 1 & 9 & - \\
\hline
\end{tabular}

$\mathrm{SD}=$ standard deviation. ${ }^{*} \alpha=0.017$ (since Bonferroni's correction $=0.05 / 3$, i.e., three comparisons); ${ }^{\mathrm{a}}$ significant $p$ value in relation to group 3 ; ${ }^{\mathrm{b}}$ significant $p$ value in relation to group 2 (Kruskal-Wallis test, Dunn's posttest); ${ }^{c} p<0.05$ in relation to group 2 , to access differences regarding complications of diabetes (Mann-Whitney test, $\alpha=5 \%$ ); some data from G1, G2, and G3 were also presented in de Souza Bastos et al. [19], Bastos et al. [20], and Corbi et al. [21].

and waist/hip proportions. This information can also be found in our previous studies [19-21].

3.2. Candidate Genes and $R T-q P C R$. Candidate genes selected to the RT-qPCR study are shown in Table 2. Thirty individuals were investigated for each gene, and after obtaining the Cts in the qPCR, we withdraw the outliers (mean of five individuals who presented huge difference regarding the group). We chose to use this approach in order to ensure that the significance was due the group and not influenced by a single individual.

In the $\mathrm{G} 1+\mathrm{G} 2$ versus $\mathrm{G} 3$ comparison, which evaluated the influence of T2D independent of glycemic control, we found that the HLA-DQA1 $(p<0.0001)$ and SOS1 $(p=0.005)$ genes were upregulated and the BRCA2 $(p=0.008)$ was downregulated. The qPCR results are presented in Figure 1(a). The full name of each gene can be found in Table 2 .

Regarding G2 versus G1, which evaluated the influence of the glycemic control in patients with T2D, the genes AGBL5 $(p=0.043), \quad C C D N 3 \quad(p=0.0003), \quad C E B P D \quad(p<0.0001)$, VAMP2 $(p=0.0001)$, ENO2 $(p<0.0001)$, and MAP2K5 $(p=0.027)$ were significantly upregulated in the G2 subjects compared to the G1 subjects (Figure 1(b)). In addition, the PPAP2B $(p=0.021)$ and LGALS12 $(p=0.0004)$ genes were downregulated (Table 2).

Comparison between G2 versus G3, which evaluated the influence of T2D with good glycemic control, showed that the HLA-DQA1 $(p=<0.0001)$ and HLA-DQB1 $(p=<0.0001)$ genes were upregulated and the MCM4 and SEC13 genes were downregulated DEGs in G2 subjects
( $p=0.0006$ and $p=0.008$, resp., Figure $1(\mathrm{c})$ ). And lastly, the comparison of $\mathrm{G} 1$ versus $\mathrm{G} 3$, which evaluated the influence of poor glycemic control of T2D patients, showed that the PPIC gene was upregulated in the G1 $(p=0.001$, Table 2, Figure 1(d)).

\section{Discussion}

Despite the knowledge in the field, the molecular processes regarding the adequate or poor glycemic control in patients with T2D remain poorly understood. Additional studies seeking to investigate DGEs in these conditions might shed some light on the molecular processes underlying it. In the present study, we investigated candidate genes potentially relevant for the glycemic control in a large cohort of patients with poorly or well-controlled T2D subjects and normoglycemic individuals. It is important to highlight that our cohort of patients was strictly selected after a complete clinical evaluation. The quality and robustness of the eligibility criteria certainly contributed to promote confident findings.

Candidate genes were chosen from a panel of DEGs obtained from microarray data (unpublished data). In order to identify DEGs in T2D, independent of the glycemic control, we compared G1 + G2 versus G3. Using this approach, we selected the $H L A-D Q A 1$ and $H L A-D Q B 1$ as candidate genes. Our results showed that both genes were upregulated in T2D patients (Figures 1(a) and 1(c)). Major histocompatibility complex (MHC), class II, is a heterodimer of molecules consisting of an alpha (DQA) and a beta chain (DQB), both anchored in the membrane. It plays a central role in the 
TABLE 2: DEGs detected by microarray chosen as good candidates for validation by RT-qPCR.

\begin{tabular}{|c|c|c|c|c|}
\hline Comparison & Gene title & Gene symbol & $\begin{array}{l}\text { Microarray FC } \\
(\text { FDR } p \text { value })\end{array}$ & $\begin{array}{c}\text { RT- } \mathrm{qPCR} \\
p \text { value (validation) } \\
\end{array}$ \\
\hline \multicolumn{5}{|l|}{$G 1+G 2$ versus $G 3$} \\
\hline${ }^{\circledR} 213831$ at \#Hs03007426 mH & $\begin{array}{c}\text { Major histocompatibility complex, } \\
\text { class II, DQ alpha } 1\end{array}$ & $H L A-D Q A 1$ & $127.80(0.002)$ & $<0.0001$ \\
\hline @212777_at \#Hs00893134_m1 & Son of sevenless homolog 1 (drosophila) & SOS1 & $2.60(0.005)$ & 0.005 \\
\hline${ }^{\circledR} 222381$ at \#Hs00918237_m1 & Aryl-hidrocarbon receptor repressor & PDCD6 & $-2.43(0.007)$ & NV \\
\hline @208368_s_at \#Hs00609073_m1 & Breast cancer 2, early onset & $B R C A 2$ & $-1.46(0.009)$ & 0.008 \\
\hline${ }^{\circledR}$ 210808_s_at \#Hs01071081_m1 & NADPH oxidase 1 & NOX1 & $-1.43(0.002)$ & NV \\
\hline \multicolumn{5}{|l|}{ G2 versus $G 1$} \\
\hline${ }^{\circledR} 231857$ s_at \#Hs01005454_g1 & ATP/GTP-binding protein-like 5 & $A G B L 5$ & $6.08(0.002)$ & 0.042 \\
\hline$@ 1562028$ at \#Hs00236949_m1 & Cyclin D3 & $\mathrm{CCDN} 3$ & $1.99(0.006)$ & 0.0003 \\
\hline 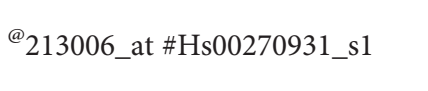 & $\begin{array}{l}\text { CCAAT/enhancer-binding } \\
\text { protein }(\mathrm{C} / \mathrm{EBP}), \text { delta }\end{array}$ & $C E B P D$ & $1.89(0.007)$ & $<0.0001$ \\
\hline @214792_x_at \#Hs00360269_m1 & Vesicle-associated membrane protein 2 & VAMP2 & $1.67(0.001)$ & 0.0001 \\
\hline${ }^{\circledR}$ 201313_at \#Hs01102367_g1 & Enolase 2 (gamma, neuronal) & ENO2 & $1.66(0.0008)$ & $<0.0001$ \\
\hline${ }^{\circledR} 211370$ s_at \#Hs00177134_m1 & Mitogen-activated protein kinase 5 & MAP2K5 & $1.39(0.001)$ & 0.027 \\
\hline${ }^{\circledR}$ 212226_s_at \#Hs00170359_m1 & Phosphatidic acid phosphatase type $2 \mathrm{~B}$ & $P P A P 2 B$ & $-2.68(0.005)$ & 0.021 \\
\hline @223828_s_at \#Hs00263821_m1 & Lectin, galactoside-binding, soluble 12 & LGALS12 & $-2.09(0.007)$ & 0.0004 \\
\hline \multicolumn{5}{|l|}{ G2 versus $G 3$} \\
\hline${ }^{\circledR} 209480$ at \#Hs03054971_m1 & $\begin{array}{c}\text { Major histocompatibility complex, } \\
\text { class II, DQ beta } 1\end{array}$ & $H L A-D Q B 1$ & $131.31(0.005)$ & $<0.0001$ \\
\hline${ }^{@} 211571$ _s_at \#Hs00171642_m1 & Versican & $V C A N$ & $1.73(0.009)$ & NV \\
\hline @212142_at \#Hs00907398_m1 & Minichromosome maintenance & MCM4 & $-4.46(0.0001)$ & 0.0006 \\
\hline @239617_at \#Hs01115007_m1 & SEC13 homolog (S. cerevisiae) & SEC13 & $-2.34(0.007)$ & 0.008 \\
\hline @205672_at \#Hs00166045_m1 & $\begin{array}{l}\text { Xeroderma pigmentosum, } \\
\text { complementation group A }\end{array}$ & $X P A$ & $-1.29(0.003)$ & NV \\
\hline \multicolumn{5}{|l|}{$G 1$ versus $G 3$} \\
\hline${ }^{\circledR} 204517$ at \#Hs00917412_m1 & $\begin{array}{c}\text { Peptidylprolyl isomerase } \\
\text { C (cyclosphilin C) }\end{array}$ & PPIC & $2.21(0.008)$ & 0.001 \\
\hline${ }^{\circledR} 1554586$ _a_at \#Hs01598095_g1 & Rho-related BTB domain containing 2 & RHOBTB2 & $2.13(0.007)$ & NV \\
\hline @1559921_at \#Hs00169777_m1 & $\begin{array}{l}\text { Platelet/endothelial cell adhesion } \\
\text { molecule (CD31 antigen) }\end{array}$ & PECAM1 & $1.86(0.004)$ & NV \\
\hline${ }^{\circledR} 215723$ _s_at \#Hs00160118_m1 & $\begin{array}{c}\text { Phospholipase D1, } \\
\text { phosphatidylcholine-specific }\end{array}$ & PLD1 & $-3.77(0.005)$ & $\mathrm{NV}$ \\
\hline
\end{tabular}

$\mathrm{FC}=$ fold change $\mathrm{FDR}$ = false discovery rate method according the Benjamini-Hochberg procedure was used for correcting each $p$ value for multiple hypothesis testing; NV = microarray not validated by RT-qPCR; ${ }^{\circledR}=$ probe set ID; \# = TaqMan code of Applied Biosystems.

immune system by presenting peptides derived from extracellular proteins. Class II molecules are expressed in antigen-presenting cells (APC: B lymphocytes, dendritic cells, and macrophages) (http://v4.genecards.org/cgi-bin/ carddisp.pl?gene $=$ HLA-DQB1\&UserNotification $=$ BetaRe direct). $H L A-D Q A 1-D Q B 1$ genes are related to leukocyte chemotaxis; G-protein signaling; IL-17-induced mucin expression; Th1, Th2, Th9, Th17, and Th22 cell differentiations; and immunological synapse formation. The current results led us to suppose that the HLA-DQA1-DQB1 overexpression in T2D patients, mainly in well-controlled diabetics (G2), could indicate that an adequate glycemic control might reduce inflammatory response, supporting the link between inflammation and glucose metabolic disturbance [22]. Interestingly, in spite of the MHC class II molecules HLA-DQA1 and $D Q B 1$ being classically associated with type $1 \mathrm{DM}$, we observed their high expression in the investigated patients with T2D. A higher frequency of the high-risk HLA$D Q B 1^{*} 0201 / 0302$ was found in patients with latent autoimmune diabetes in adults (LADA) [23]. Also, a study comprised of 695 families with patients affected by type 1 and others by type $2 \mathrm{DM}$ (mixed families) had HLA class II risk haplotypes, such as DR3(17)-DQA1*0501-DQB1*02. This result supports a possible genetic interaction between type 1 and type 2 diabetes mediated by the HLA locus [24].

Patients with T2D (G1 + G2) showed an upregulation of the SOS1 in comparison to the normoglycemic individuals (G3). The SOS1 gene is related to immune response pathways, such as IL-7 signaling in B lymphocytes, regulation of lipid metabolism, and the insulin signaling pathway [25]. 
$\mathrm{G} 1+\mathrm{G} 2$ versus $\mathrm{G} 3$
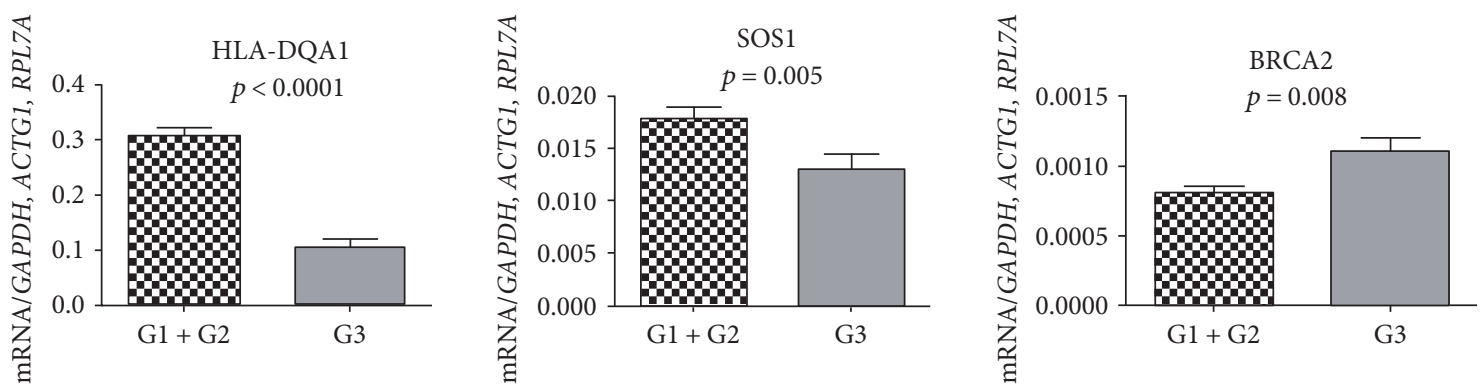

(a)

G2 versus $G 1$
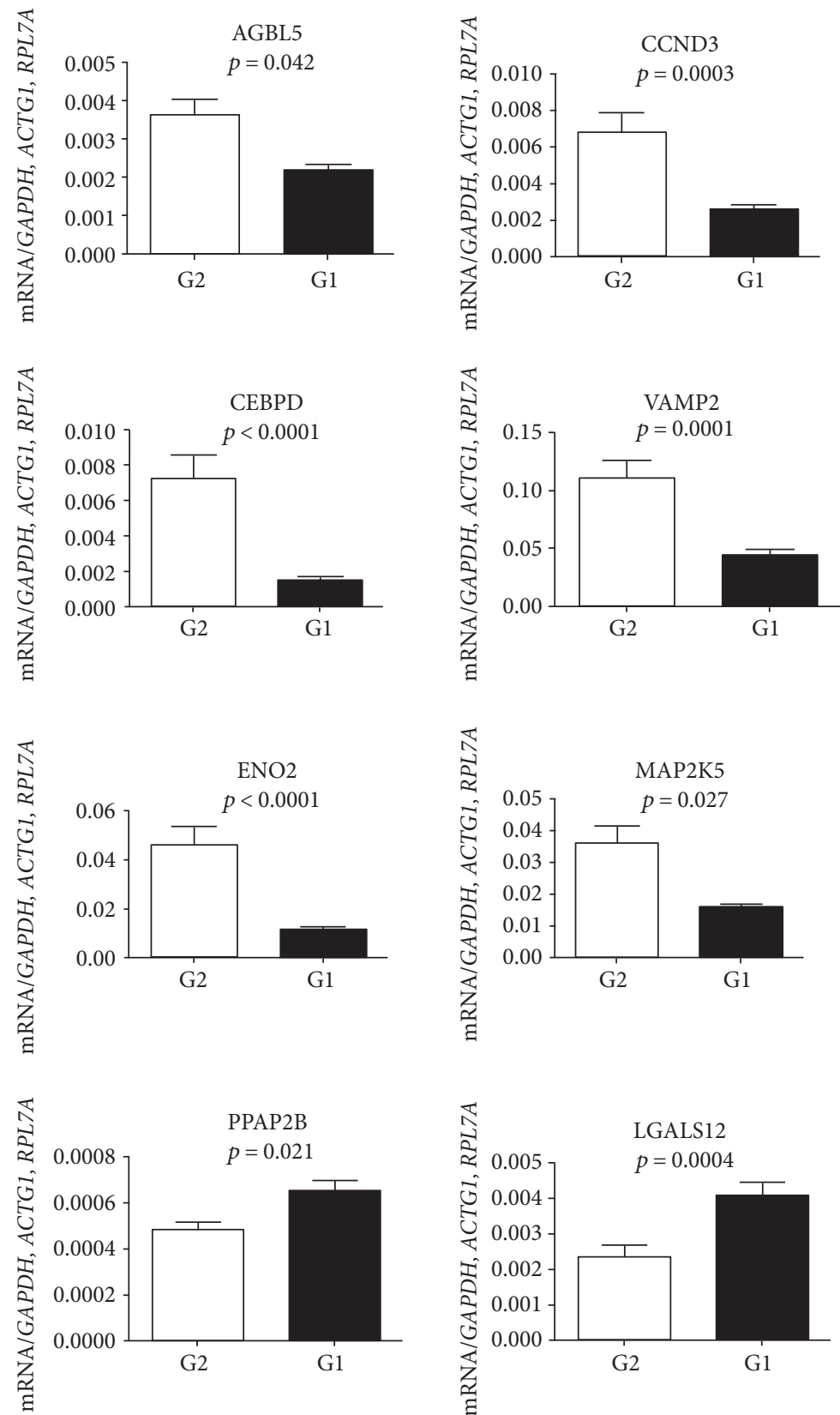

(b)

FIgUre 1: Continued. 
G2 versus G3
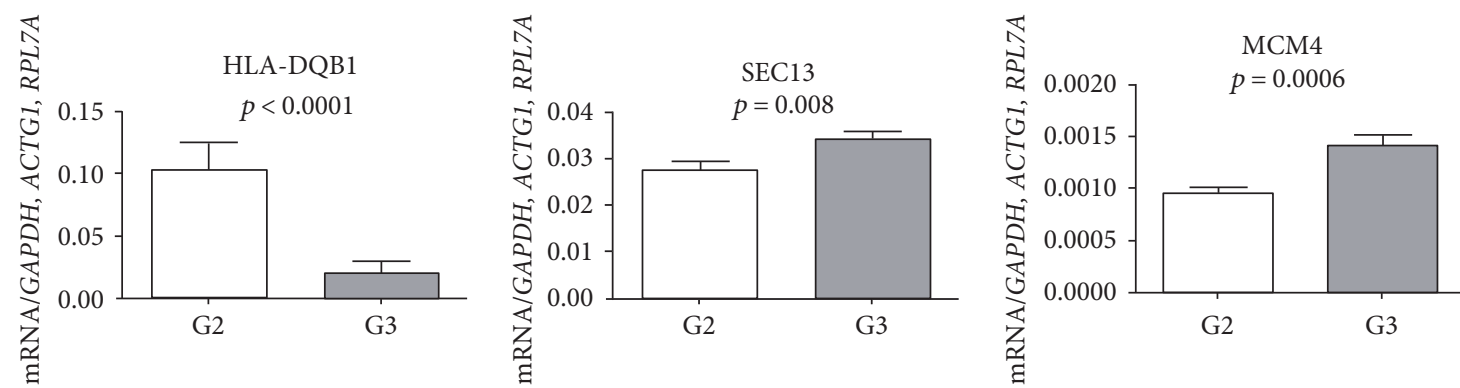

(c)

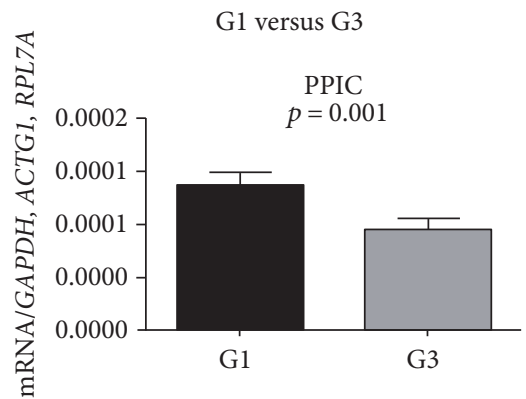

(d)

Figure 1: Validation results by RT-qPCR of the genes considering the different comparisons. (a) G1 + G2 versus G3; (b) G2 versus G1; (c) G1 versus G3; (d) G2 versus G3. All mRNA levels of the investigated genes were normalized to a mean of the endogenous controls GAPDH, $A C T G 1$, and RPL7A genes. Data represent the mean \pm SEM of 30 patients per group (Mann-Whitney $U$ test; $\alpha=5 \%$ ). $p$ values are presented in Table 2.

Myers and colleagues described that during insulin stimulation, insulin receptor-expressing cells result in the tyrosine phosphorylation of SHC-transforming protein 1 or insulin receptor substrate 1 (IRS-1), which are both associated with growth factor receptor-bound protein 2 (GRB-2). GRB-2 activates p2l-RAS by forming a stable complex through its SH3 domains to SOS-1 [25]. Moreover, the SOS1 gene is involved in the regulation of lipid metabolism and insulin signaling generic cascades (http://lsresearch.thomsonreuters .com/maps/724). It is worth bearing in mind that all patients are similarly affected by dyslipidemia; therefore, it is reasonable to infer that the presence of T2D in the G1 + G2 groups could have an additive effect for the higher expression of SOS1 in comparison to the G3 normoglycemic patients.

In contrast, the BRCA2 gene was downregulated in G1 + G2 compared to G3. This result is interesting because this gene is related to the repair of the damage to the double-stranded DNA pathway indicating that T2D patients may have a deficiency in this process. This is in agreement with Corbi et al. [21], who found higher irreversible damage to the double-stranded DNA in T2D patients than in those without the disease (G3) by utilizing the micronucleus test in the same patients enrolled in the present study.

Considering the well-controlled diabetics (G2) as reference, we found out that the CCDN3, CEBPD, and MAP2K5 genes were upregulated in G2 when compared to G1 (poorly controlled diabetics) (Figure 1(b)). Taking into mind, the gene functions are as follows: (i) CCDN3 gene is associated with the IL-5 immune-related response and with IGF-1 (growth factor similar to insulin-like 1) receptor signaling; (ii) CEBPD gene is associated with the IL-6 and IL-17 signaling pathways and; (iii) MAP2K5 gene is related to T lymphocytes and the inflammatory response; it seems to indicate that the well-controlled diabetics (G2) could better orchestrate the immune system functions. Also, we found an overexpression of the enolase 2 (ENO2, alias NSE) and vesicle-associated membrane protein 2 (VAMP2) genes in G2 subjects. The function of the VAMP2 gene is linked to the secretion of insulin by pancreatic $\beta$-cells. The VAMP- 2 protein is also associated with the regulation of GLUT-4 trafficking and fusion in adipocytes. In animals with diabetes induced by streptozotocin and nicotinamide, resveratrol supplementation increased the VAMP2 gene expression and blood insulin level, as well as reduced the fasting blood glucose and improved the insulin resistance [26]. Because we validated here higher expression of the VAMP2 gene in the G2 group, it seems to indicate that the good glycemic control contributes to increase the insulin production in these patients, in comparison to G1 poorly controlled T2D patients. Obviously, it is essential to T2D patients to achieve and maintain the glycemic control, preferentially without adding basal insulin therapy, because it was related to increased number of patients experiencing hypoglycemia during 1 year of follow-up of basal insulin therapy [27]. Interestingly, intensive glycemic control was associated with higher rates of severe hypoglycemia than less intensive control, as raised by the ACCORD (Action 
to Control Cardiovascular Risk in Diabetes), ADVANCE (Action in Diabetes and Vascular Disease: Preterax and Diamicron MR Controlled Evaluation), and VADT (Veterans Affairs Diabetes Trial) studies [28-30].

Another DEG in the G2 versus G1 comparison is the $E N O 2$, which encodes an intracellular protein present mainly in the neuronal cytoplasm, in central and peripheral neuroendocrine cells $[31,32]$, and in white blood cells (http://www.genecards.org/cgi-bin/carddisp.pl?gene=ENO2\& keywords=ENO2). Interestingly, ENO2 is also associated with the glycolysis and gluconeogenesis processes (http:// lsresearch.thomsonreuters.com/maps/9300). ENO2 or NSE is readily secreted into the cerebrospinal fluid and blood after tissue injury and is related to diabetic retinopathy $[31,33]$. Retinopathy, neuropathy, and nephropathy are common complications in patients with diabetes mellitus. Early diabetic retinopathy includes a neurodegenerative component, and diabetic retinopathy is generally viewed as a neurovascular disease [33]. Circulating ENO2/NSE mRNA levels were lower in diabetics with neuropathy (mean $\mathrm{HbAlc}=8.2 \%$ ) than in those without neuropathy (mean $\mathrm{HbAlc}=6.6 \%$ ) [31]. Here, we also found significant lower levels of circulating ENO2 mRNA levels in G1 (mean HbA1c $=10.4 \%$, poorly controlled T2D) than in G2 (mean $\mathrm{HbAlc}=6.6 \%$, wellcontrolled T2D) (Figure 1(b)). We noticed from the 30 patients in each group that retinopathy affected 6 patients in the G1 and 1 patient in the G2. Retinopathy was also related to the poor glycemic control of T2D [34].

Still comparing G2 versus G1, the LGALS12 (lectin, galactoside-binding, soluble 12) gene was upregulated in G1. Genome-wide association studies (GWAS) available at: http://www.gwascentral.org/study accessed (Aug. 24, 2016) demonstrated the LGALS12 gene as risk loci of BMI (HGVST308) and is associated with glycemic traits (HGVST463). In spite of the validation of $P P A P 2 B$ and $A G B L 5$ genes, demonstrated $p$ values are not significant after Bonferroni's correction, and if we consider that it is not necessary to correct for multiple tests to obtain $p$ values of qPCR analysis because each gene was independently investigated, we can assume that these genes were also validated. Whether we consider this, our results are in line with GWAS studies which associated $P P A P 2 B$ gene with T2D (HGVST3; HGVST5), glycated hemoglobin levels (HGVST618), and glycemic traits (HGVST463), while the AGBL5 was associated with GWAS of glycemic traits (HGVST463).

When comparing the well-controlled T2D (G2) patients versus normoglycemics (G3), besides the aforementioned HLA-DQB1 gene, we found that SEC13 and MCM4 genes presented downregulation in both microarray and RTqPCR. Interestingly, SEC13 and MCM4 genes were also associated with GWAS of glycemic traits (HGVST463) and glycated hemoglobin (HGVST618). MCM4 participated in the DNA replication in the early $\mathrm{S}$ phase, and altered expression and methylation of MCM4 gene were recently observed in the zebrafish model of metabolic memory (MM). This was defined as the persistence of diabetic (DM) complications even after glycemic control was pharmacologically achieved [35]. According to Leontovich et al., the results were consistent with human diabetic epigenetic studies and provided one explanation for the persistence of long-term tissue complications as seen in diabetes [35]. Furthermore, in agreement with the lower levels of MCM4 gene in the PBMC of well-controlled T2D (G2) patients (Figure 1(c)), decreased expression of MCM4, leading to decreased DNA replication of vascular smooth muscle cells, was demonstrated [36]. This occurs by the overexpression of PGC- $1 \beta$, which is upregulated by metformin. Therefore, a functional role of MCM4 gene was presented in the context of vascular injury commonly found in diabetes [36].

Lastly, comparing G1 versus G3, we found an upregulation of the PPIC gene in G1. PPIC gene was associated with endometrial, kidney, and lung neoplasms. Some studies have demonstrated an increase of malignant neoplasms in T2D patients $[37,38]$. In addition, the PPIC gene was associated with GWAS of glycemic traits (HGVST463) and glycated hemoglobin (HGVST618).

By using the candidate genes/RT-qPCR approach in a well-evaluated cohort, we presented some DEGs in welland poorly controlled T2D patients. Although our findings are relevant in the context of T2D, it is important to discuss that the present study has some limitations. We agree that there is a lack of a longitudinal study of data tracking of diseases in our patients, and the blood analysis was made in a unique time point, making it difficult and harming the best diagnosis of a T2D patient as well as the poorly controlled. In the present study, we did not assess the genes at the translational level; that is, we did not quantify the proteins encoded by the genes investigated here. Certainly, further studies could clinically follow up the patients enrolled here and could focus on the translational levels of the genes investigated in the present study. Proteomics studies could ascertain the present mRNA findings or reveal different results, since the gene regulation comprises very complex processes. Because the expression profiling was investigated in PBMC, we cannot exclude the possibility that gene expression could be influenced by proportions of the blood cell types in the circulation, as inferred by Miranda et al. [39]. However, we assessed the amount of lymphocytes, neutrophils, eosinophils, and monocytes in each patient investigated here and they were statistically similar among groups, demonstrating that our findings were not influenced by a specific cell type.

Despite the mentioned limitations, this study brings functional information related to glycemic control on gene expression, which was related to the immune response and regulation of glycemic and lipid metabolism, as well as DNA replication and damage. Even though the present study is not the first evidence of the majority association of genes investigated here with $\mathrm{T} 2 \mathrm{D}$, they could be thought as more newness in the association with glycemic metabolism than others, such as the HLA-DQA1, HLA-DQB1, VAMP2, and ENO genes.

We concluded that some candidate genes potentially associated with adequate glycemic control in T2D patients were validated. Moreover, this study contributed to the knowledge of how the glycemic control could systemically influence gene expression. Further case-control studies and meta-analysis approach with strict clinical selection of larger 
and ethnic diverse populations should be made to confirm and strengthen our results.

\section{Conflicts of Interest}

The authors declare no conflicts of interest.

\section{Authors' Contributions}

Sâmia Cruz Tfaile Corbi, Alliny Souza Bastos, Raquel Mantuaneli Scarel-Caminaga, Silvana Regina Perez Orrico, and Claudia V. Maurer-Morelli conceived and designed the experiments. Sâmia Cruz Tfaile Corbi, Rafael Nepomuceno, and Thamiris Cirelli performed the experiments. Sâmia Cruz Tfaile Corbi, Raquel Mantuaneli Scarel-Caminaga, Silvana Regina Perez Orrico, Claudia V. Maurer-Morelli, and Cristiane S. Rocha analyzed the data. Sâmia Cruz Tfaile Corbi, Raquel Mantuaneli Scarel-Caminaga, Claudia V. Maurer-Morelli, Raquel Alves dos Santos, and Catarina Satie Takahashi contributed reagents/materials/analysis tools. Sâmia Cruz Tfaile Corbi, Raquel Mantuaneli ScarelCaminaga, Silvana Regina Perez Orrico, and Claudia V. Maurer-Morelli wrote the paper.

\section{Acknowledgments}

This study was supported by the Foundation for Research Support of State of São Paulo (FAPESP), Grants 2007/ 08362-8, 2009/16233-9, and 2010/10882-2, and the Coordination of Improvement of Higher Education Students of the Brazilian Ministry of Education (CAPES). The authors thank Maria Eugenia R. Camargo, for her technical support with the methodology of microarray, as well as the Brazilian Center for Research in Energy and Materials (CNPEM) and the Brazilian Biosciences National Laboratory (LNBio) for supplying the equipment to perform the microarray methodology.

\section{References}

[1] G. M. Zuniga-Gonzalez, C. M. Batista-Gonzalez, B. C. GomezMeda et al., "Micronuclei in diabetes: folate supplementation diminishes micronuclei in diabetic patients but not in an animal model," Mutation Research, vol. 634, no. 1-2, pp. 126-134, 2007.

[2] P. M. Duarte, J. P. Bezerra, T. S. Miranda, M. Feres, L. Chambrone, and L. M. Shaddox, "Local levels of inflammatory mediators in uncontrolled type 2 diabetic subjects with chronic periodontitis," Journal of Clinical Periodontology, vol. 41, no. 1, pp. 11-18, 2014.

[3] E. Tatsch, G. V. Bochi, S. J. Piva et al., "Association between DNA strand breakage and oxidative, inflammatory and endothelial biomarkers in type 2 diabetes," Mutation Research, vol. 732, no. 1-2, pp. 16-20, 2012.

[4] Y. Kataoka, M. Shao, K. Wolski et al., "Multiple risk factor intervention and progression of coronary atherosclerosis in patients with type 2 diabetes mellitus," European Journal of Preventive Cardiology, vol. 20, no. 2, pp. 209-217, 2013.
[5] A. D. Mooradian, "Dyslipidemia in type 2 diabetes mellitus," Nature Clinical Practice Endocrinology \& Metabolism, vol. 5, no. 3, pp. 150-159, 2009.

[6] M. Soory, "Inflammatory mechanisms and redox status in periodontal and cardiometabolic diseases: effects of adjunctive nutritional antioxidants and statins," Infectious Disorders Drug Targets, vol. 12, no. 4, pp. 301-315, 2012.

[7] A. M. Iacopino, "Periodontitis and diabetes interrelationships: role of inflammation," Annals of Periodontology/the American Academy of Periodontology, vol. 6, no. 1, pp. 125-137, 2001.

[8] C. W. Cutler, E. A. Shinedling, M. Nunn et al., "Association between periodontitis and hyperlipidemia: cause or effect?" Journal of Periodontology, vol. 70, no. 12, pp. 1429-1434, 1999.

[9] P. O. Nassar, C. S. Walker, C. S. Salvador, F. A. Felipetti, S. R. Orrico, and C. A. Nassar, "Lipid profile of people with diabetes mellitus type 2 and periodontal disease," Diabetes Research and Clinical Practice, vol. 96, no. 1, pp. 35-39, 2012.

[10] J. Almeida Abdo, F. R. Cirano, M. Z. Casati et al., "Influence of dyslipidemia and diabetes mellitus on chronic periodontal disease," Journal of Periodontology, vol. 84, no. 10, pp. 14011408, 2013.

[11] A. Pacilli, S. D. Cosmo, V. Trischitta, and S. Bacci, "Role of relationship between $\mathrm{HbAlc}$, fibrinogen and HDLcholesterol on cardiovascular disease in patients with type 2 diabetes mellitus," Atherosclerosis, vol. 228, no. 1, pp. 247-248, 2013.

[12] C. W. Cutler, R. L. Machen, R. Jotwani, and A. M. Iacopino, "Heightened gingival inflammation and attachment loss in type 2 diabetics with hyperlipidemia," Journal of Periodontology, vol. 70, no. 11, pp. 1313-1321, 1999.

[13] F. Ismail-Beigi, T. Craven, M. A. Banerji et al., "Effect of intensive treatment of hyperglycaemia on microvascular outcomes in type 2 diabetes: an analysis of the ACCORD randomised trial," Lancet, vol. 376, no. 9739, pp. 419-430, 2010.

[14] ACCORD Study Group, ACCORD Eye Study Group, E. Y. Chew et al., "Effects of medical therapies on retinopathy progression in type 2 diabetes," The New England Journal of Medicine, vol. 363, no. 3, pp. 233-244, 2010.

[15] J. Feng, Y. Liu, L. M. Chu et al., "Changes in microvascular reactivity after cardiopulmonary bypass in patients with poorly controlled versus controlled diabetes," Circulation, vol. 126, no. 11, pp. 73-80, 2012.

[16] F. S. Manoel-Caetano, D. J. Xavier, A. F. Evangelista et al., "Gene expression profiles displayed by peripheral blood mononuclear cells from patients with type 2 diabetes mellitus focusing on biological processes implicated on the pathogenesis of the disease," Gene, vol. 511, no. 2, pp. 151-160, 2012.

[17] P. C. Tsiotra, C. Tsigos, E. Yfanti et al., "Visfatin, TNF-alpha and IL-6 mRNA expression is increased in mononuclear cells from type 2 diabetic women," Hormone and Metabolic Research $=$ Hormon- und Stoffwechselforschung $=$ Hormones et Metabolisme, vol. 39, no. 10, pp. 758-763, 2007.

[18] K. Gokulakrishnan, K. T. Mohanavalli, F. Monickaraj, V. Mohan, and M. Balasubramanyam, "Subclinical inflammation/oxidation as revealed by altered gene expression profiles in subjects with impaired glucose tolerance and type 2 diabetes patients," Molecular and Cellular Biochemistry, vol. 324, no. 1-2, pp. 173-181, 2009.

[19] A. de Souza Bastos, D. T. Graves, A. P. d. Melo Loureiro et al., "Diabetes and increased lipid peroxidation are associated with systemic inflammation even in well-controlled patients," 
Journal of Diabetes and Its Complications, vol. 30, no. 8, pp. 1593-1599, 2016.

[20] A. S. Bastos, D. T. Graves, A. P. Loureiro et al., "Lipid peroxidation is associated with the severity of periodontal disease and local inflammatory markers in patients with type 2 diabetes," The Journal of Clinical Endocrinology and Metabolism, vol. 97, no. 8, pp. 1353-1362, 2012.

[21] S. C. Corbi, A. S. Bastos, S. R. Orrico et al., "Elevated micronucleus frequency in patients with type 2 diabetes, dyslipidemia and periodontitis," Mutagenesis, vol. 29, no. 6, pp. 433-439, 2014.

[22] I. Vinagre, J. L. Sanchez-Quesada, J. Sanchez-Hernandez et al., "Inflammatory biomarkers in type 2 diabetic patients: effect of glycemic control and impact of LDL subfraction phenotype," Cardiovascular Diabetology, vol. 13, no. 1, p. 34, 2014.

[23] T. Tuomi, A. Carlsson, H. Li et al., "Clinical and genetic characteristics of type 2 diabetes with and without GAD antibodies," Diabetes, vol. 48, no. 1, pp. 150-157, 1999.

[24] H. Li, E. Lindholm, P. Almgren et al., "Possible human leukocyte antigen-mediated genetic interaction between type 1 and type 2 diabetes," The Journal of Clinical Endocrinology and Metabolism, vol. 86, no. 2, pp. 574-582, 2001.

[25] M. G. Myers Jr., L. M. Wang, X. J. Sun et al., "Role of IRS-1GRB-2 complexes in insulin signaling," Molecular and Cellular Biology, vol. 14, no. 6, pp. 3577-3587, 1994.

[26] A. Rezaei Farimani, M. Saidijam, M. T. Goodarzi et al., "Effect of resveratrol supplementation on the SNARE proteins expression in adipose tissue of stroptozotocin-nicotinamide induced type 2 diabetic rats," Iranian Journal of Medical Sciences, vol. 40, no. 3, pp. 248-255, 2015.

[27] V. Fonseca, E. Chou, H. W. Chung, and C. Gerrits, "Economic burden of hypoglycemia with basal insulin in type 2 diabetes," The American Journal of Managed Care, vol. 23, no. 2, pp. 114-122, 2017.

[28] Action to Control Cardiovascular Risk in Diabetes Study Group, H. C. Gerstein, M. E. Miller et al., "Effects of intensive glucose lowering in type 2 diabetes," The New England Journal of Medicine, vol. 358, no. 24, pp. 2545-2559, 2008.

[29] ADVANCE Collaborative Group, A. Patel, S. MacMahon et al., "Intensive blood glucose control and vascular outcomes in patients with type 2 diabetes," The New England Journal of Medicine, vol. 358, no. 24, pp. 2560-2572, 2008.

[30] W. Duckworth, C. Abraira, T. Moritz et al., "Glucose control and vascular complications in veterans with type 2 diabetes," The New England Journal of Medicine, vol. 360, no. 2, pp. 129-139, 2009.

[31] H. S. Sandhu, A. N. Butt, J. Powrie, and R. Swaminathan, "Measurement of circulating neuron-specific enolase mRNA in diabetes mellitus," Annals of the New York Academy of Sciences, vol. 1137, pp. 258-263, 2008.

[32] P. J. Marangos and D. E. Schmechel, "Neuron specific enolase, a clinically useful marker for neurons and neuroendocrine cells," Annual Review of Neuroscience, vol. 10, pp. 269-295, 1987.

[33] J. Li, M. Yan, Y. Zhang, M. Xie, L. Yan, and J. Chen, "Serum neuron-specific enolase is elevated as a novel indicator of diabetic retinopathy including macular oedema," Diabetic Medicine : A Journal of the British Diabetic Association, vol. 32, no. 1, pp. 102-107, 2015.

[34] S. G. Kim, J. R. Hahm, D. K. Kim, S. R. Cho, and D. S. Choi, "Assessment of glycemic control in patients with type 2 diabetes mellitus treated with metformin-sulfonylurea combination: results of a multicenter, cross-sectional, observational study in Korea," Journal of Diabetes Investigation, vol. 6, no. 3, pp. 317-324, 2015.

[35] A. A. Leontovich, R. V. Intine, and M. P. Sarras Jr., "Epigenetic studies point to DNA replication/repair genes as a basis for the heritable nature of long term complications in diabetes," Journal of Diabetes Research, vol. 2016, Article ID 2860780, 10 pages, 2016.

[36] T. Guo, L. Landsman, N. Li, and M. Hebrok, "Factors expressed by murine embryonic pancreatic mesenchyme enhance generation of insulin-producing cells from hESCs," Diabetes, vol. 62, no. 5, pp. 1581-1592, 2013.

[37] C. Li and D. Kong, "Cancer risks from diabetes therapies: evaluating the evidence," Pharmacology \& Therapeutics, vol. 144, no. 1, pp. 71-81, 2014.

[38] A. Sekikawa, H. Fukui, T. Maruo, T. Tsumura, Y. Okabe, and Y. Osaki, "Diabetes mellitus increases the risk of early gastric cancer development," European Journal of Cancer, vol. 50, no. 12, pp. 2065-2071, 2014.

[39] D. N. Miranda, D. K. Coletta, L. J. Mandarino, and G. Q. Shaibi, "Increases in insulin sensitivity among obese youth are associated with gene expression changes in whole blood," Obesity, vol. 22, no. 5, pp. 1337-1344, 2014. 


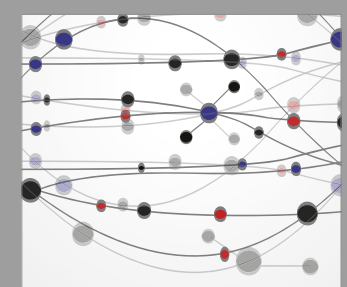

The Scientific World Journal
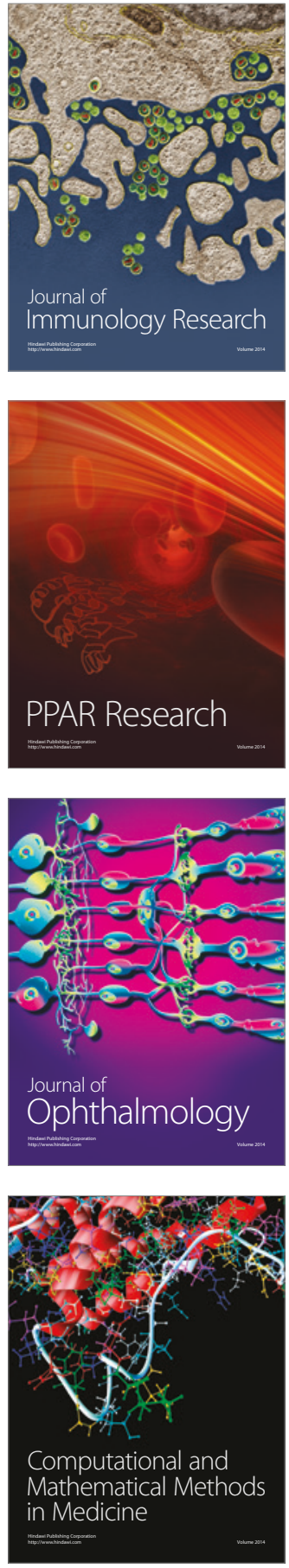

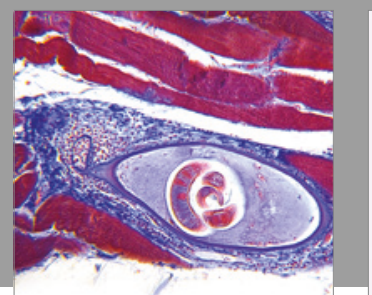

Gastroenterology Research and Practice
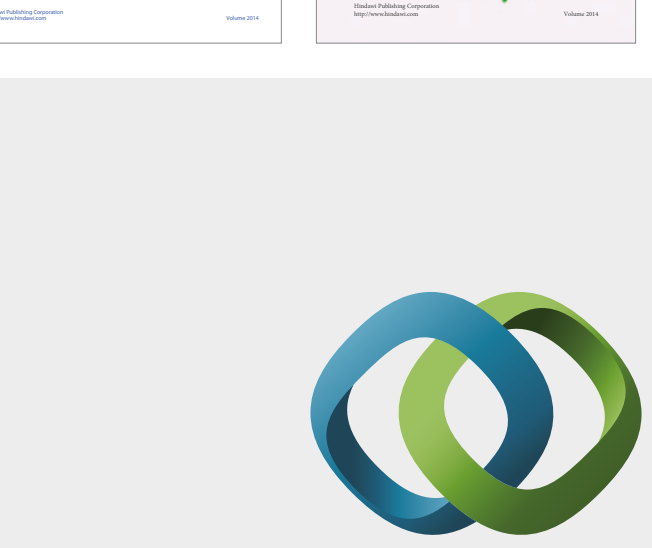

\section{Hindawi}

Submit your manuscripts at

https://www.hindawi.com
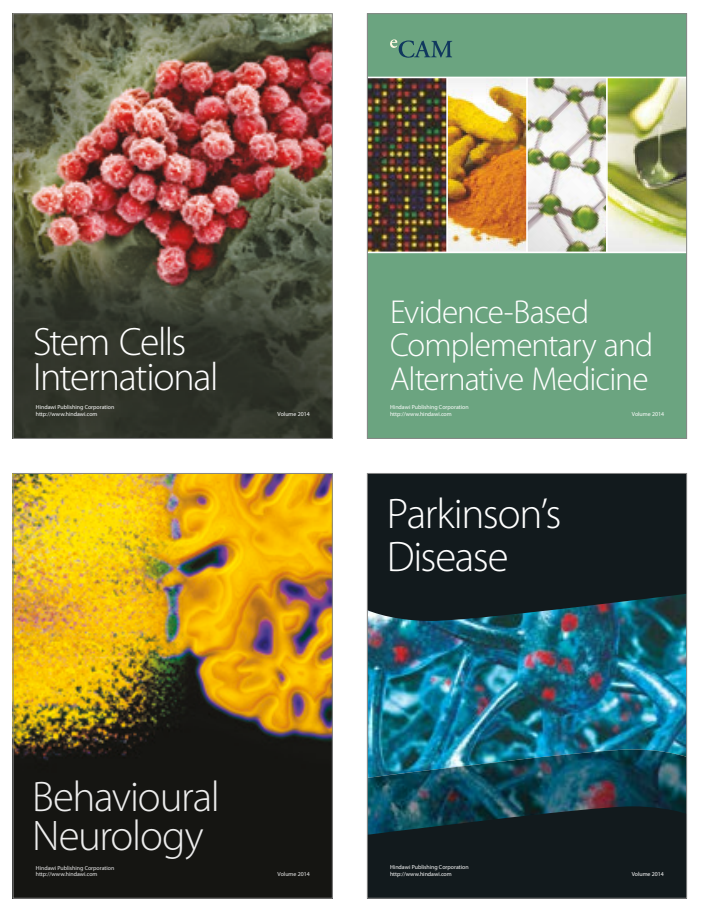
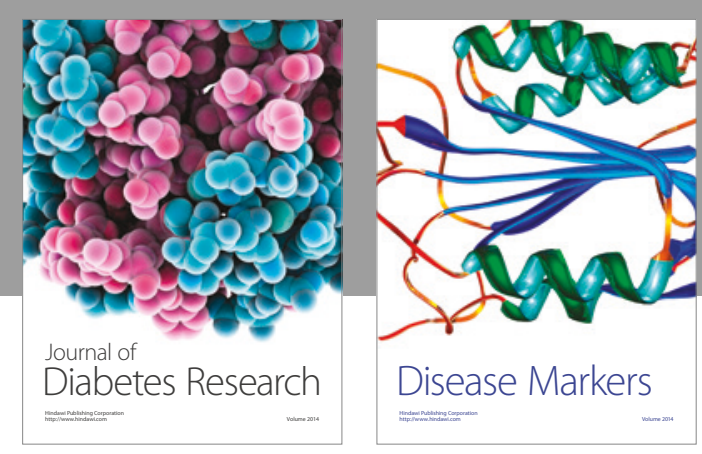

Disease Markers
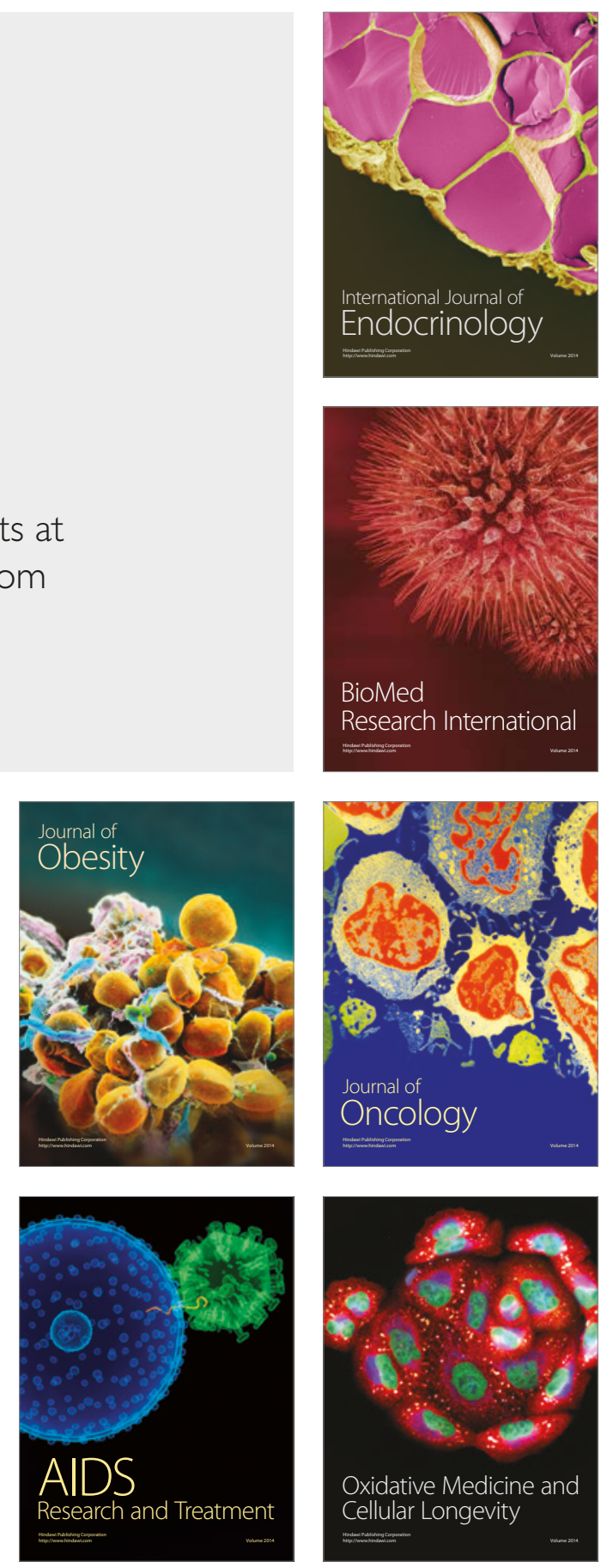